\title{
Bounds on some new weakly singular Wendroff-type integral inequalities and applications
}

\section{Kelong Zheng*}

\section{"Correspondence:}

zhengkelong@swust.edu.cn School of Science, Southwest University of Science and Technology, Mianyang, Sichuan 621010, P.R. China

\begin{abstract}
With a more concise condition for the ordered parameter groups, some nonlinear weakly singular integral inequalities of Wendroff type, which generalize some existing results, are established. Furthermore, application examples in the boundedness and uniqueness of the solution of a singular partial integral equation are given.
\end{abstract}

MSC: 26A33; 34A08; 34A34; 45J05

Keywords: integral inequalities; weakly singular; Wendroff type; boundedness

\section{Introduction}

The Gronwall-Bellman integral inequality and its various nonlinear versions have made great achievements in the qualitative analysis for the solutions of differential and integral equations, as shown in [1,2] and [3]. However, qualitative properties and numerical analysis for the solutions of singular integral and differential equations depend on the study of corresponding integral inequalities, and the aforementioned inequalities are not directly applicable. In recent years, many researchers have devoted much effort to investigating weakly singular integral inequalities and their applications (see [4-12]). For example, the Volterra-type singular integro-differential equation

$$
\begin{aligned}
& y^{\prime}(t)=f(t, y(t))+c \int_{0}^{t} \frac{y^{\prime}(s)}{(t-s)^{\alpha}} d s+q(t), \\
& y(0)=y_{0}, \quad t \in[0, T], T>0,
\end{aligned}
$$

was discussed by McKee [8] when $\alpha=\frac{1}{2}$ for the diffusion of discrete particles in a turbulent fluid; Henry [13] proposed a linear integral inequality with singular kernel to investigate some qualitative properties for a parabolic differential equation; Sano and $\mathrm{Ku}$ nimatsu [14] gave a modified version of the Henry-type inequality; Ye et al. [15] used a generalized inequality to study the dependence of the solution for a fractional differential equation. In particular, Medved' [16] presented a new method to discuss nonlinear singular integral inequalities of Henry type and generalized his results to analogue Wendroff inequalities for functions in two variables. A slightly improved de-singularity approach has been used by Ma and Yang [6] to study a more general singular integral inequality as

(c) 2013 Zheng; licensee Springer. This is an Open Access article distributed under the terms of the Creative Commons Attribution License (http://creativecommons.org/licenses/by/2.0), which permits unrestricted use, distribution, and reproduction in any medium, provided the original work is properly cited. 
follows:

$$
\begin{aligned}
u(t) \leq & a(t)+b(t) \int_{0}^{t}\left(t^{\sigma}-s^{\sigma}\right)^{\mu-1} s^{\gamma-1} g(s) u(s) d s \\
& +c(t) \int_{0}^{t}\left(t^{\sigma}-s^{\sigma}\right)^{\mu-1} s^{\gamma-1} g(s) w(u(s)) d s .
\end{aligned}
$$

Later Ma and Pečarić [17] used this method to establish a priori bounds on solutions to the following nonlinear singular integral inequalities with power nonlinearity:

$$
u^{p}(t) \leq a(t)+b(t) \int_{0}^{t}\left(t^{\alpha}-s^{\alpha}\right)^{\beta-1} s^{\gamma-1} f(s) u^{q}(s) d s, \quad t \in R_{+} .
$$

Bounds on solutions to inequalities (1.2) and (1.3) are established for the cases when the ordered parameter groups $[\alpha, \beta, \gamma]$ and $[\sigma, \mu, \tau]$ obey distribution I or II (for details, see [6]).

With the development of the theory of inequalities for a two-dimensional case, more attention has also been paid to weakly singular integral inequalities in two variables and their applications to the partial differential equation with singular kernel. Upon the results in [6] and [17], Cheung and Ma [18] investigated some new weakly singular integral inequalities of Wendroff type

$$
\begin{aligned}
u^{p}(x, y) \leq & a(x, y)+b(x, y) \int_{0}^{x} \int_{0}^{y}\left(x^{\alpha}-s^{\alpha}\right)^{\beta-1} s^{\gamma-1}\left(y^{\alpha}-t^{\alpha}\right)^{\beta-1} t^{\gamma-1} \\
& \times f(s, t) u^{q}(s, t) d s d t,
\end{aligned}
$$

and their more general nonlinear version was presented by Wang and Zheng [19]. It is to be noted that the ordered parameter groups $[\alpha, \beta, \gamma]$ in [18] and [19] make the application of inequalities more inconvenient. To overcome the weakness, in this paper we avoid the above-mentioned conditions and use another concise assumption to discuss some more general integral inequalities of Wendroff type. Our estimates are quite simple and the resulting formulas are similar to the classical Gronwall-Bihari inequalities. Furthermore, to show the applications of these inequalities, some examples are presented.

\section{Main result}

In what follows, $R$ denotes the set of real numbers and $R_{+}=(0, \infty) . C(X, Y)$ denotes the collection of continuous functions from the set $X$ to the set $Y . D_{1} z(x, y)$ and $D_{2} z(x, y)$ denote the first-order partial derivatives of $z(x, y)$ with respect to $x$ and $y$, respectively.

Lemma 2.1 (Discrete Jensen inequality) Let $A_{1}, A_{2}, \ldots, A_{n}$ be nonnegative real numbers and $r>1$ be a real number. Then

$$
\left(A_{1}+A_{2}+\cdots+A_{n}\right)^{r} \leq n^{r-1}\left(A_{1}^{r}+A_{2}^{r}+\cdots+A_{n}^{r}\right) .
$$

Lemma 2.2 (see [6]) Let $\alpha, \beta, \gamma$ and $p$ be positive constants. Then

$$
\int_{0}^{t}\left(t^{\alpha}-s^{\alpha}\right)^{p(\beta-1)} s^{p(\gamma-1)} d s=\frac{t^{\theta}}{\alpha} B\left[\frac{p(\gamma-1)+1}{\alpha}, p(\beta-1)+1\right], \quad t \in R_{+},
$$


where $B[\xi, \eta]=\int_{0}^{1} s^{\xi-1}(1-s)^{\eta-1} d s(\operatorname{Re} \xi>0, \operatorname{Re} \eta>0)$ is the well-known B-function and $\theta=p[\alpha(\beta-1)+\gamma-1]+1$.

Firstly, consider the following Wendroff-type integral inequality:

$$
\begin{aligned}
u(x, y) \leq & a(x, y)+\int_{0}^{x} \int_{0}^{y}\left(x^{\alpha_{1}}-s^{\alpha_{1}}\right)^{\beta_{1}-1} s^{\gamma_{1}-1}\left(y^{\alpha_{2}}-t^{\alpha_{2}}\right)^{\beta_{2}-1} t^{\gamma_{2}-1} \\
& \times f(x, y, s, t) w(u(s, t)) d s d t .
\end{aligned}
$$

The basic assumptions for inequality (2.1) are as follows:

$\left(\mathrm{A}_{1}\right) \alpha_{i} \in(0,1], \beta_{i} \in(0,1)$ and $\gamma_{i}>1-\frac{1}{p}$ such that $\frac{1}{p}+\alpha_{i}\left(\beta_{i}-1\right)+\gamma_{i}-1 \geq 0(p>1, i=1,2)$;

$\left(\mathrm{A}_{2}\right) a(x, y) \in C\left(R_{+}^{2}, R_{+}\right)$and $f(x, y, s, t) \in C\left(R_{+}^{4}, R_{+}\right)$;

$\left(\mathrm{A}_{3}\right) w(u) \in C\left(R_{+}, R_{+}\right)$is nondecreasing and $w(0)=0$.

Let $\tilde{a}(x, y)=\max _{0 \leq \tau \leq x, 0 \leq \eta \leq y} a(\tau, \eta)$ and $\tilde{f}(x, y, s, t)=\max _{0 \leq \tau \leq x, 0 \leq \eta \leq y} f(\tau, \eta, s, t)$.

Theorem 2.1 Under assumptions $\left(\mathrm{A}_{1}\right),\left(\mathrm{A}_{2}\right)$ and $\left(\mathrm{A}_{3}\right)$, if $u(x, y) \in C\left(R_{+}^{2}, R+\right)$ satisfies $(2.1)$, then

$$
u(x, y) \leq\left[W_{1}^{-1}\left(W_{1}(A(x, y))+2^{q-1}\left(M_{1} x^{\theta_{1}} M_{2} y^{\theta_{2}}\right)^{\frac{q}{p}} \int_{0}^{x} \int_{0}^{y} \tilde{f}^{q}(x, y, s, t) d s d t\right)\right]^{\frac{1}{q}}
$$

for $0 \leq x \leq X_{1}$ and $0 \leq y \leq Y_{1}$, where

$$
\begin{aligned}
& A(x, y)=2^{q-1} \tilde{a}^{q}(x, y), \\
& M_{i}=\frac{1}{\alpha_{i}} B\left[\frac{p\left(\gamma_{i}-1\right)+1}{\alpha_{i}}, p\left(\beta_{i}-1\right)+1\right], \\
& \theta_{i}=p\left[\alpha_{i}\left(\beta_{i}-1\right)+\gamma_{i}-1\right]+1, \quad i=1,2,
\end{aligned}
$$

$\frac{1}{p}+\frac{1}{q}=1, W_{1}^{-1}$ is the inverse of $W_{1}$,

$$
W_{1}(u)=\int_{u_{0}}^{u} \frac{d \xi}{w^{q}\left(\xi^{\frac{1}{q}}\right)}, \quad u \geq u_{0}>0,
$$

and $X_{1}, Y_{1} \in R_{+}$are chosen such that

$$
W_{1}(A(x, y))+2^{q-1}\left(M_{1} x^{\theta_{1}} M_{2} y^{\theta_{2}}\right)^{\frac{q}{p}} \int_{0}^{x} \int_{0}^{y} \tilde{f}^{q}(x, y, s, t) d s d t \in \operatorname{Dom}\left(W_{1}^{-1}\right) .
$$

Proof By the definition of $\tilde{a}(x, y)$ and $\tilde{f}(x, y, s, t)$, it is easy to see that $\tilde{a}(x, y)$ and $\tilde{f}(x, y, s, t)$ are nonnegative and nondecreasing in $x$ and $y$. Moreover, $\tilde{a}(x, y) \geq a(x, y)$ and $\tilde{f}(x, y, s, t) \geq$ $f(x, y, s, t)$. From (2.1), it follows that

$$
\begin{aligned}
u(x, y) \leq & \tilde{a}(x, y)+\int_{0}^{x} \int_{0}^{y}\left(x^{\alpha_{1}}-s^{\alpha_{1}}\right)^{\beta_{1}-1} s^{\gamma_{1}-1}\left(y^{\alpha_{2}}-t^{\alpha_{2}}\right)^{\beta_{2}-1} t^{\gamma_{2}-1} \\
& \times \tilde{f}(x, y, s, t) w(u(s, t)) d s d t .
\end{aligned}
$$


According to the assumption $\left(\mathrm{A}_{1}\right)$, we choose suitable indices $p$, $q$. Applying the Hölder inequality with indices $p, q$ to (2.6), we get

$$
\begin{aligned}
u(x, y) \leq & \tilde{a}(x, y)+\left(\int_{0}^{x} \int_{0}^{y}\left(x^{\alpha_{1}}-s^{\alpha_{1}}\right)^{p\left(\beta_{1}-1\right)} s^{p\left(\gamma_{1}-1\right)}\left(y^{\alpha_{2}}-t^{\alpha_{2}}\right)^{p\left(\beta_{2}-1\right)} t^{p\left(\gamma_{2}-1\right)} d s d t\right)^{\frac{1}{p}} \\
& \times\left(\int_{0}^{x} \int_{0}^{y} \tilde{f}^{q}(x, y, s, t) w^{q}(u(s, t)) d s d t\right)^{\frac{1}{q}} .
\end{aligned}
$$

By Lemma 2.1, from (2.7), we have

$$
\begin{aligned}
& u^{q}(x, y) \\
& \leq 2^{q-1}\left[\tilde{a}^{q}(x, y)+\left(\int_{0}^{x} \int_{0}^{y}\left(x^{\alpha_{1}}-s^{\alpha_{1}}\right)^{p\left(\beta_{1}-1\right)} s^{p\left(\gamma_{1}-1\right)}\left(y^{\alpha_{2}}-t^{\alpha_{2}}\right)^{p\left(\beta_{2}-1\right)} t^{p\left(\gamma_{2}-1\right)} d s d t\right)^{\frac{q}{p}}\right. \\
& \left.\quad \times\left(\int_{0}^{x} \int_{0}^{y} \tilde{f}^{q}(x, y, s, t) w^{q}(u(s, t)) d s d t\right)\right] \\
& =2^{q-1} \tilde{a}^{q}(x, y)+2^{q-1}\left(M_{1} x^{\theta_{1}} M_{2} y^{\theta_{2}}\right)^{\frac{q}{p}} \\
& \quad \times\left(\int_{0}^{x} \int_{0}^{y} \tilde{f}^{q}(x, y, s, t) w^{q}(u(s, t)) d s d t\right)
\end{aligned}
$$

where $M_{i}$ and $\theta_{i}$ are given by (2.3) for $i=1,2$.

Since $p, q>0$ and $\theta_{i} \geq 0(i=1,2), \tilde{a}(x, y)^{q}$ and $\left(x^{\theta_{1}} y^{\theta_{2}}\right)^{\frac{q}{p}}$ are also nondecreasing in $x$ and $y$. Taking any arbitrary $\tilde{x}$ and $\tilde{y}$ with $\tilde{x} \leq X_{1}, \tilde{y} \leq Y_{1}$, we obtain

$$
\begin{aligned}
u^{q}(x, y) \leq & 2^{q-1} \tilde{a}^{q}(\tilde{x}, \tilde{y})+2^{q-1}\left(M_{1} \tilde{x}^{\theta_{1}} M_{2} \tilde{y}^{\theta^{2}}\right)^{\frac{q}{p}} \\
& \times\left(\int_{0}^{x} \int_{0}^{y} \tilde{f}^{q}(\tilde{x}, \tilde{y}, s, t) w^{q}(u(s, t)) d s d t\right)
\end{aligned}
$$

for $0 \leq x \leq \tilde{x}, 0 \leq y \leq \tilde{y}$. Let

$$
A(\tilde{x}, \tilde{y})=2^{q-1} \tilde{a}^{q}(\tilde{x}, \tilde{y})
$$

and

$$
z(x, y)=A(\tilde{x}, \tilde{y})+2^{q-1}\left(M_{1} \tilde{x}^{\theta_{1}} M_{2} \tilde{y}^{\theta_{2}}\right)^{\frac{q}{p}}\left(\int_{0}^{x} \int_{0}^{y} \tilde{f}^{q}(\tilde{x}, \tilde{y}, s, t) w^{q}(u(s, t)) d s d t\right) .
$$

Then $u^{q}(x, y) \leq z(x, y)$, namely $u(x, y) \leq z^{\frac{1}{q}}(x, y)$. Meanwhile, $z(0, y)=A(\tilde{x}, \tilde{y})$. Considering

$$
\begin{aligned}
D_{1} z(x, y) & =2^{q-1}\left(M_{1} \tilde{x}^{\theta_{1}} M_{2} \tilde{y}^{\theta_{2}}\right)^{\frac{q}{p}}\left(\int_{0}^{y} \tilde{f}^{q}(\tilde{x}, \tilde{y}, x, t) w^{q}(u(x, t)) d t\right) \\
& \leq 2^{q-1}\left(M_{1} \tilde{x}^{\theta_{1}} M_{2} \tilde{y}^{\theta_{2}}\right)^{\frac{q}{p}}\left(\int_{0}^{y} \tilde{f}^{q}(\tilde{x}, \tilde{y}, x, t) w^{q}\left(z^{\frac{1}{q}}(x, t)\right) d t\right) \\
& \leq 2^{q-1}\left(M_{1} \tilde{x}^{\theta_{1}} M_{2} \tilde{y}^{\theta_{2}}\right)^{\frac{q}{p}} w^{q}\left(z^{\frac{1}{q}}(x, y)\right)\left(\int_{0}^{y} \tilde{f}^{q}(\tilde{x}, \tilde{y}, x, t) d t\right),
\end{aligned}
$$


where we apply the fact that $w^{q}\left(z^{\frac{1}{q}}(x, y)\right)$ is nondecreasing in $y$, we have

$$
\frac{D_{1} z(x, y)}{w^{q}\left(z^{\frac{1}{q}}(x, y)\right)} \leq 2^{q-1}\left(M_{1} \tilde{x}^{\theta_{1}} M_{2} \tilde{y}^{\theta_{2}}\right)^{\frac{q}{p}} \int_{0}^{y} \tilde{f}^{q}(\tilde{x}, \tilde{y}, x, t) d t .
$$

Integrating both sides of the above inequality from 0 to $x$, we obtain

$$
\begin{aligned}
W_{1}(z(x, y)) & \leq W_{1}(z(0, y))+2^{q-1}\left(M_{1} \tilde{x}^{\theta_{1}} M_{2} \tilde{y}^{\theta_{2}}\right)^{\frac{q}{p}} \int_{0}^{x} \int_{0}^{y} \tilde{f}^{q}(\tilde{x}, \tilde{y}, s, t) d s d t \\
& =W_{1}(A(\tilde{x}, \tilde{y}))+2^{q-1}\left(M_{1} \tilde{x}^{\theta_{1}} M_{2} \tilde{y}^{\theta_{2}}\right)^{\frac{q}{p}} \int_{0}^{x} \int_{0}^{y} \tilde{f}^{q}(\tilde{x}, \tilde{y}, s, t) d s d t
\end{aligned}
$$

for $0 \leq x \leq \tilde{x}, 0 \leq y \leq \tilde{y}$, where $W_{1}(u)$ is given by (2.4). By virtue of the assumption $\left(\mathrm{A}_{3}\right)$, $W_{1}(u)$ is strictly increasing. So, its inverse $W_{1}^{-1}$ is continuous and increasing in its corresponding domain. Replacing $x$ and $y$ by $\tilde{x}$ and $\tilde{y}$, we have

$$
W_{1}(z(\tilde{x}, \tilde{y})) \leq W_{1}(A(\tilde{x}, \tilde{y}))+2^{q-1}\left(M_{1} \tilde{x}^{\theta_{1}} M_{2} \tilde{y}^{\theta_{2}}\right)^{\frac{q}{p}} \int_{0}^{\tilde{x}} \int_{0}^{\tilde{y}} \tilde{f}^{q}(\tilde{x}, \tilde{y}, s, t) d s d t .
$$

Since $\tilde{x}$ and $\tilde{y}$ are arbitrary, we replace $\tilde{x}$ and $\tilde{y}$ by $x$ and $y$, respectively, and get

$$
W_{1}(z(x, y)) \leq W_{1}(A(x, y))+2^{q-1}\left(M_{1} x^{\theta_{1}} M_{2} y^{\theta_{2}}\right)^{\frac{q}{p}} \int_{0}^{x} \int_{0}^{y} \tilde{f}^{q}(x, y, s, t) d s d t
$$

for $0 \leq x \leq X_{1}$ and $0 \leq y \leq Y_{1}$. The above inequality can be rewritten as

$$
z(x, y) \leq W_{1}^{-1}\left(W_{1}(A(x, y))+2^{q-1}\left(M_{1} x^{\theta_{1}} M_{2} y^{\theta_{2}}\right)^{\frac{q}{p}} \int_{0}^{x} \int_{0}^{y} \tilde{f}^{q}(x, y, s, t) d s d t\right) .
$$

Therefore, by $u(x, y) \leq z^{\frac{1}{q}}(x, y),(2.2)$ holds for $0 \leq x \leq X_{1}$ and $0 \leq y \leq Y_{1}$.

Remark 2.1 If we take $\alpha_{1}=\alpha_{2}=\alpha, \beta_{1}=\beta_{2}=\beta$ and $\gamma_{1}=\gamma_{2}=\gamma$, respectively, weakly singular kernel in (2.1) reduces to the formula in (1.4). Especially, it should be pointed out that if $\alpha_{i} \in(0,1], \beta_{i} \in\left(\frac{1}{2}, 1\right)$ and $p>2$, the ordered parameter group is just I type defined in [6], and if $\alpha_{i} \in(0,1], \beta_{i} \in\left(0, \frac{1}{2}\right)$ and $p>3$, it is just II type defined in [6].

Remark 2.2 In [20], Medved' has investigated the Wendroff inequality with weakly singular kernel $(x-s)^{\alpha-1}(y-t)^{\beta-1}$. However, his result holds under the condition ' $\alpha>\frac{1}{2}, \beta>\frac{1}{2}$ ' and the assumption that ' $w(u)$ satisfies the condition $(q)$ ' (see Definition 2.1 in [20]). In our result, the condition $(q)$ is eliminated, and the ordered parameter groups can be discussed in more cases.

Corollary 2.1 Under assumptions $\left(\mathrm{A}_{1}\right),\left(\mathrm{A}_{2}\right)$ and $\left(\mathrm{A}_{3}\right)$, let $\lambda>0, \mu>0(\lambda>\mu)$. If $u(x, y) \in$ $C\left(R_{+}^{2}, R+\right)$ satisfies

$$
\begin{aligned}
u^{\lambda}(x, y) \leq & a(x, y)+\int_{0}^{x} \int_{0}^{y}\left(x^{\alpha_{1}}-s^{\alpha_{1}}\right)^{\beta_{1}-1} s^{\gamma_{1}-1}\left(y^{\alpha_{2}}-t^{\alpha_{2}}\right)^{\beta_{2}-1} t^{\gamma_{2}-1} \\
& \times f(x, y, s, t) u^{\mu}(s, t) d s d t,
\end{aligned}
$$


then

$$
\begin{aligned}
u(x, y) \leq & {\left[\left(2^{q-1} \tilde{a}(x, y)\right)^{\frac{\lambda-\mu}{\lambda}}+\frac{\lambda-\mu}{\lambda} 2^{q-1}\left(M_{1} x^{\theta_{1}} M_{2} y^{\theta_{2}}\right)^{\frac{q}{p}}\right.} \\
& \left.\times \int_{0}^{x} \int_{0}^{y} \tilde{f}^{q}(x, y, s, t) d s d t\right]^{\frac{1}{(\lambda-\mu) q}}
\end{aligned}
$$

for $x \geq 0$ and $y \geq 0$, where $p, q, M_{i}$ and $\theta_{i}(i=1,2)$ are defined as in Theorem 2.1.

Proof Let $v(x, y)=u^{\lambda}(x, y)$, then $u(x, y)=v^{\frac{1}{\lambda}}(x, y)$, that is, $u^{\mu}(x, y)=v^{\frac{\mu}{\lambda}}(x, y)$. It follows from (2.12) that

$$
v(x, y) \leq a(x, y)+\int_{0}^{x} \int_{0}^{y}\left(x^{\alpha_{1}}-s^{\alpha_{1}}\right)^{\beta_{1}-1} s^{\gamma_{1}-1}\left(y^{\alpha_{2}}-t^{\alpha_{2}}\right)^{\beta_{2}-1} t^{\gamma_{2}-1} f(x, y, s, t) v^{\frac{\mu}{\lambda}}(s, t) d s d t .
$$

Clearly, $w(v)=v^{\frac{\mu}{\lambda}}$ satisfies the assumption $\left(\mathrm{A}_{3}\right)$. By the definition of $W_{1}(u)$ by $(2.4)$, letting $u_{0}=0$, we have

$$
W_{1}(u)=\int_{0}^{u} \frac{d \xi}{\xi^{\frac{\mu}{\lambda}}}=\frac{\lambda}{\lambda-\mu} u^{\frac{\lambda-\mu}{\lambda}}
$$

and

$$
W_{1}^{-1}(u)=\left(\frac{\lambda-\mu}{\lambda} u\right)^{\frac{\lambda}{\lambda-\mu}}, \quad \operatorname{Dom}\left(W_{1}^{-1}\right)=[0, \infty)
$$

Substituting (2.14) and (2.15) into (2.2), we get

$$
v(x, y) \leq\left[\left(2^{q-1} \tilde{a}(x, y)\right)^{\frac{\lambda-\mu}{\lambda}}+\frac{\lambda-\mu}{\lambda} 2^{q-1}\left(M_{1} x^{\theta_{1}} M_{2} y^{\theta_{2}}\right)^{\frac{q}{p}} \int_{0}^{x} \int_{0}^{y} \tilde{f}^{q}(x, y, s, t) d s d t\right]^{\frac{\lambda}{(\lambda-\mu) q}} .
$$

In view of $u(x, y)=v^{\frac{1}{\lambda}}(x, y)$, we can obtain (2.13).

Remark 2.3 Let $\lambda=2$ and $\mu=1$; we can get the interesting Henry-Ou-Iang type singular integral inequality in two variables. As for the concrete formula, we omit it here.

Now we turn to consider the case $\lambda=\mu$. In fact, (2.12) can be reduced to the corresponding linear version. Hence we only need to prove the following result.

Corollary 2.2 Under assumptions $\left(\mathrm{A}_{1}\right),\left(\mathrm{A}_{2}\right)$ and $\left(\mathrm{A}_{3}\right)$, if $u(x, y) \in C\left(R_{+}^{2}, R+\right)$ satisfies

$$
\begin{aligned}
u(x, y) \leq & a(x, y)+\int_{0}^{x} \int_{0}^{y}\left(x^{\alpha_{1}}-s^{\alpha_{1}}\right)^{\beta_{1}-1} s^{\gamma_{1}-1}\left(y^{\alpha_{2}}-t^{\alpha_{2}}\right)^{\beta_{2}-1} t^{\gamma_{2}-1} \\
& \times f(x, y, s, t) u(s, t) d s d t
\end{aligned}
$$

then

$$
u(x, y) \leq 2^{\frac{q-1}{q}} \tilde{a}(x, y) \exp \left(\frac{2^{q-1}}{q}\left(M_{1} x^{\theta_{1}} M_{2} y^{\theta_{2}}\right)^{\frac{q}{p}} \int_{0}^{x} \int_{0}^{y} \tilde{f}^{q}(x, y, s, t) d s d t\right)
$$

for $x \geq 0$ and $y \geq 0$, where $p, q, M_{i}$ and $\theta_{i}(i=1,2)$ are defined as in Theorem 2.1. 
Proof In (2.17), $w(u)=u$ also satisfies the assumption $\left(\mathrm{A}_{3}\right)$. Here, we have

$$
W_{1}(u)=\int_{u_{0}}^{u} \frac{d \xi}{\xi}=\ln \frac{u}{u_{0}}, \quad W_{1}^{-1}(u)=u_{0} \exp (u), \quad \operatorname{Dom}\left(W_{1}^{-1}\right)=[0, \infty) .
$$

Similar to the computation in Corollary 2.1, the estimate (2.17) holds.

Next, we consider the more complicated Wendroff-type integral inequality

$$
\begin{aligned}
u(x, y) \leq & a(x, y)+\int_{0}^{x} \int_{0}^{y} f_{1}(x, y, s, t) w_{1}(u(s, t)) d s d t \\
& +\int_{0}^{x} \int_{0}^{y}\left(x^{\alpha_{1}}-s^{\alpha_{1}}\right)^{\beta_{1}-1} s^{\gamma_{1}-1}\left(y^{\alpha_{2}}-t^{\alpha_{2}}\right)^{\beta_{2}-1} t^{\gamma_{2}-1} \\
& \times f_{2}(x, y, s, t) w_{2}(u(s, t)) d s d t .
\end{aligned}
$$

Note that the nonsingular case $f_{2}(x, y, s, t)=0$ of the above integral inequality was studied by Pachpatte [21].

Theorem 2.2 Under the assumption $\left(\mathrm{A}_{1}\right)$, suppose that $w_{1}$ and $w_{2}$ satisfy the assumption $\left(\mathrm{A}_{3}\right)$. If $a(x, y) \in C\left(R_{+}^{2}, R_{+}\right), f_{1}(x, y, s, y), f_{2}(x, y, s, t) \in C\left(R_{+}^{4}, R_{+}\right)$and $u(x, y) \in C\left(R_{+}^{2}, R+\right)$ satisfies (2.19), then

$$
\begin{aligned}
u(x, y) \leq & {\left[W _ { 2 } ^ { - 1 } \left(W_{2}\left(3^{q-1} \tilde{a}^{q}(x, y)\right)+3^{q-1}(x y)^{\frac{q}{p}} \int_{0}^{x} \int_{0}^{y} \tilde{f}_{1}^{q}(x, y, s, t) d s d t\right.\right.} \\
& \left.\left.+3^{q-1}\left(M_{1} x^{\theta_{1}} M_{2} y^{\theta_{2}}\right)^{\frac{q}{p}} \int_{0}^{x} \int_{0}^{y} \tilde{f}_{2}^{q}(x, y, s, t) d s d t\right)\right]^{\frac{1}{q}}
\end{aligned}
$$

for $0 \leq x \leq X_{2}$ and $0 \leq y \leq Y_{2}$, where $p, q, M_{i}$ and $\theta_{i}(i=1,2)$ are defined as in Theorem 2.1, $W_{2}(u)$ is defined by

$$
W_{2}(u)=\int_{u_{0}}^{u} \frac{d \xi}{w_{1}^{q}\left(\xi^{\frac{1}{q}}\right)+w_{2}^{q}\left(\xi^{\frac{1}{q}}\right)}, \quad u \geq u_{0}>0
$$

and $X_{2}, Y_{2} \in R_{+}$are chosen such that

$$
\begin{aligned}
& W_{2}\left(3^{q-1} \tilde{a}^{q}(x, y)\right)+3^{q-1}(x y)^{\frac{q}{p}} \int_{0}^{x} \int_{0}^{y} \tilde{f}_{1}^{q}(x, y, s, t) d s d t \\
& +3^{q-1}\left(M_{1} x^{\theta_{1}} M_{2} y^{\theta_{2}}\right)^{\frac{q}{p}} \int_{0}^{x} \int_{0}^{y} \tilde{f}_{2}^{q}(x, y, s, t) d s d t \in \operatorname{Dom}\left(W_{2}^{-1}\right) .
\end{aligned}
$$

Proof As in the proof of Theorem 2.1, it follows from (2.19) that

$$
\begin{aligned}
u(x, y) \leq & \tilde{a}(x, y)+\int_{0}^{x} \int_{0}^{y} \tilde{f}_{1}(x, y, s, t) w_{1}(u(s, t)) d s d t \\
& +\int_{0}^{x} \int_{0}^{y}\left(x^{\alpha_{1}}-s^{\alpha_{1}}\right)^{\beta_{1}-1} s^{\gamma_{1}-1}\left(y^{\alpha_{2}}-t^{\alpha_{2}}\right)^{\beta_{2}-1} t^{\gamma_{2}-1} \\
& \times \tilde{f}_{2}(x, y, s, t) w_{2}(u(s, t)) d s d t .
\end{aligned}
$$


Noticing that

$$
\begin{aligned}
& \int_{0}^{x} \int_{0}^{y} \tilde{f}_{1}(x, y, s, t) w_{1}(u(s, t)) d s d t \\
& \quad \leq\left(\int_{0}^{x} \int_{0}^{y} 1 d s d t\right)^{\frac{1}{p}}\left(\int_{0}^{x} \int_{0}^{y} \tilde{f}_{1}^{q}(x, y, s, t) w_{1}^{q}(u(s, t)) d s d t\right)^{\frac{1}{q}},
\end{aligned}
$$

from Lemma 2.1 and Lemma 2.2, we have

$$
\begin{aligned}
u^{q}(x, y) \leq & 3^{q-1}\left[\tilde{a}^{q}(x, y)+(x y)^{\frac{q}{p}} \int_{0}^{x} \int_{0}^{y} \tilde{f}_{1}^{q}(x, y, s, t) w_{1}^{q}(u(s, t)) d s d t\right. \\
& \left.+\left(M_{1} x^{\theta_{1}} M_{2} y^{\theta_{2}}\right)^{\frac{q}{p}} \int_{0}^{x} \int_{0}^{y} \tilde{f}_{2}^{q}(x, y, s, t) w_{2}^{q}(u(s, t)) d s d t\right] .
\end{aligned}
$$

We introduce $\tilde{x}, \tilde{y}$ as in the proof of Theorem 2.1 , the above inequality can be rewritten as

$$
\begin{aligned}
u^{q}(x, y) \leq & 3^{q-1} \tilde{a}^{q}(\tilde{x}, \tilde{y})+3^{q-1}(\tilde{x} \tilde{y})^{\frac{q}{p}} \int_{0}^{x} \int_{0}^{y} \tilde{f}_{1}^{q}(\tilde{x}, \tilde{y}, s, t) w_{1}^{q}(u(s, t)) d s d t \\
& +3^{q-1}\left(M_{1} \tilde{x}^{\theta_{1}} M_{2} \tilde{y}^{\theta_{2}}\right)^{\frac{q}{p}} \int_{0}^{x} \int_{0}^{y} \tilde{f}_{2}^{q}(\tilde{x}, \tilde{y}, s, t) w_{2}^{q}(u(s, t)) d s d t .
\end{aligned}
$$

Denoting the right-hand side of $(2.25)$ by $z(x, y)$, we have

$$
u^{q}(x, y) \leq z(x, y), \quad z(0, y)=3^{q-1} \tilde{a}^{q}(\tilde{x}, \tilde{y})
$$

and

$$
\begin{aligned}
D_{1} z(x, y) \leq & 3^{q-1}(\tilde{x} \tilde{y})^{\frac{q}{p}} \int_{0}^{y} \tilde{f}_{1}^{q}(\tilde{x}, \tilde{y}, x, t) w_{1}^{q}\left(z^{\frac{1}{q}}(x, t)\right) d t \\
& +3^{q-1}\left(M_{1} \tilde{x}^{\theta_{1}} M_{2} \tilde{y}^{\theta_{2}}\right)^{\frac{q}{p}} \int_{0}^{y} \tilde{f}_{2}^{q}(\tilde{x}, \tilde{y}, x, t) w_{2}^{q}\left(z^{\frac{1}{q}}(x, t)\right) d t,
\end{aligned}
$$

which implies

$$
\begin{aligned}
\frac{D_{1} z(x, y)}{w_{1}^{q}\left(z^{\frac{1}{q}}(x, y)\right)+w_{2}^{q}\left(z^{\frac{1}{q}}(x, y)\right)} \leq & 3^{q-1}(\tilde{x} \tilde{y})^{\frac{q}{p}} \int_{0}^{y} \tilde{f}_{1}^{q}(\tilde{x}, \tilde{y}, x, t) d t \\
& +3^{q-1}\left(M_{1} \tilde{x}^{\theta_{1}} M_{2} \tilde{y}^{\theta_{2}}\right)^{\frac{q}{p}} \int_{0}^{y} \tilde{f}_{2}^{q}(\tilde{x}, \tilde{y}, x, t) d t .
\end{aligned}
$$

Similar to the proof of Theorem 2.1, we obtain (2.20). The details are omitted here.

\section{Applications}

In this section, we present some examples to show applications in the boundedness and uniqueness of a certain partial integral equation with weakly singular kernel.

Example 1 Suppose $u(x, y) \in C\left(R_{+}^{2}, R_{+}\right)$satisfies the inequality as follows:

$$
u(x, y) \leq \frac{1}{2}+\int_{0}^{x} \int_{0}^{y}(x-s)^{-\frac{1}{5}} s^{-\frac{1}{2}}(y-t)^{-\frac{1}{3}} t^{-\frac{1}{3}} e^{-s-2 t} \sqrt{u(s, t)} d s d t
$$


for $x \geq 0, y \geq 0$. Then (3.1) is the special case of inequality (2.1), that is,

$$
\begin{aligned}
& a(x, y)=\frac{1}{2}, \quad \alpha_{1}=1, \quad \beta_{1}=\frac{4}{5}, \quad \gamma_{1}=\frac{1}{2} ; \\
& \alpha_{2}=1, \quad \beta_{2}=\frac{2}{3}, \quad \gamma_{2}=\frac{2}{3} ; \\
& f(x, y, s, t)=e^{-s-2 t}, \quad w(u)=\sqrt{u(s, t)} .
\end{aligned}
$$

From the condition $\gamma_{1}>1-\frac{1}{p}$, we have $1<p<2$. From $\gamma_{2}>1-\frac{1}{p}$, we have $1<p<3$. With the assumption $\left(\mathrm{A}_{1}\right)$, let $p=\frac{4}{3}$, then $q=4, \frac{q}{p}=3$. Hence

$$
\begin{aligned}
& \tilde{a}(x, y)=\frac{1}{2}, \quad \tilde{f}(x, y, s, t)=e^{-s-2 t}, \quad A(x, y)=2^{3}\left(\frac{1}{2}\right)^{4}=\frac{1}{2} ; \\
& M_{1}=B\left[\frac{1}{3}, \frac{11}{15}\right], \quad \theta_{1}=\frac{1}{15}, \quad M_{2}=B\left[\frac{5}{9}, \frac{5}{9}\right], \quad \theta_{2}=\frac{1}{9} ; \\
& W_{1}(u)=\int_{u_{0}}^{u} \frac{d \xi}{\sqrt{\xi}}=2\left(\sqrt{u}-\sqrt{u_{0}}\right), \\
& W_{1}^{-1}(u)=\left(\sqrt{u_{0}}+\frac{u}{2}\right)^{2}, \quad \operatorname{Dom}\left(W_{1}^{-1}\right)=[0,+\infty) .
\end{aligned}
$$

Using (2.2) in Theorem 2.1, we get for $x \geq 0$ and $y \geq 0$,

$$
\begin{aligned}
u(x, y) \leq & {\left[W_{1}^{-1}\left(W_{1}(A(x, y))+2^{3}\left(M_{1} x^{\theta_{1}} M_{2} y^{\theta_{2}}\right)^{3} \int_{0}^{x} \int_{0}^{y}\left(e^{-s-2 t}\right)^{4} d s d t\right)\right]^{\frac{1}{4}} } \\
= & {\left[W _ { 1 } ^ { - 1 } \left(W_{1}\left(\frac{1}{2}\right)\right.\right.} \\
& \left.\left.+8\left(B\left[\frac{1}{3}, \frac{11}{15}\right] x^{\frac{1}{15} B}\left[\frac{5}{9}, \frac{5}{9}\right] y^{\frac{1}{9}}\right)^{3} \int_{0}^{x} \int_{0}^{y} e^{-4 s} e^{-8 t} d s d t\right)\right]^{\frac{1}{4}} \\
= & {\left[W _ { 1 } ^ { - 1 } \left(\sqrt{2}-2 \sqrt{u_{0}}\right.\right.} \\
& \left.\left.+\frac{1}{4}\left(B\left[\frac{1}{3}, \frac{11}{15}\right] x^{\frac{1}{15}} B\left[\frac{5}{9}, \frac{5}{9}\right] y^{\frac{1}{9}}\right)^{3}\left(1-e^{-4 x}\right)\left(1-e^{-8 y}\right)\right)\right]^{\frac{1}{4}} \\
= & {\left[\frac{\sqrt{2}}{2}+\frac{1}{8}\left(B\left[\frac{1}{3}, \frac{11}{15}\right] x^{\frac{1}{15}} B\left[\frac{5}{9}, \frac{5}{9}\right] y^{\frac{1}{9}}\right)^{3}\left(1-e^{-4 x}\right)\left(1-e^{-8 y}\right)\right]^{\frac{1}{2}} }
\end{aligned}
$$

which implies that $u(x, y)$ in (3.1) is bounded.

Example 2 Consider the following linear singular integral equations:

$$
u(x, y)=a(x, y)+\int_{0}^{x} \int_{0}^{y}(x-s)^{\beta_{1}-1} s^{\gamma_{1}-1}(y-t)^{\beta_{2}-1} t^{\gamma_{2}-1} f(s, t) u(s, t) d s d t
$$

and

$$
v(x, y)=b(x, y)+\int_{0}^{x} \int_{0}^{y}(x-s)^{\beta_{1}-1} s^{\gamma_{1}-1}(y-t)^{\beta_{2}-1} t^{\gamma_{2}-1} f(s, t) v(s, t) d s d t
$$


where $u, v, a, b, f \in C\left(R_{+}^{2}, R_{+}\right)$with $|a(x, y)-b(x, y)|<\epsilon$. Here, $\epsilon$ is an arbitrary positive number, and $\beta_{i}$ and $\gamma_{i}(i=1,2)$ satisfy the assumption $\left(\mathrm{A}_{1}\right)$. From (3.4) and (3.5), we get

$$
\begin{aligned}
& |u(x, y)-v(x, y)| \\
& \quad \leq|a(x, y)-b(x, y)| \\
& \quad+\int_{0}^{x} \int_{0}^{y}(x-s)^{\beta_{1}-1} s^{\gamma_{1}-1}(y-t)^{\beta_{2}-1} t^{\gamma_{2}-1} f(s, t)|u(s, t)-v(s, t)| d s d t,
\end{aligned}
$$

which is the formula of inequality (2.16). Applying Corollary 2.2 to (3.6), we have

$$
|u(x, y)-v(x, y)| \leq 2^{\frac{q-1}{q}} \epsilon \exp \left(\frac{2^{q-1}}{q}\left(M_{1} x^{\theta_{1}} M_{2} y^{\theta_{2}}\right)^{\frac{q}{p}} \int_{0}^{x} \int_{0}^{y} f(s, t)^{q} d s d t\right)
$$

where $M_{i}$ and $\theta_{i}(i=1,2)$ are defined as in Theorem 2.1. If $a(x, y)=b(x, y)$, letting $\epsilon \rightarrow 0$, we obtain the uniqueness of the global continuous solution of equation (3.4).

\section{Competing interests}

The author declares that they have no competing interests.

\section{Authors' contributions}

This work is supported by the Doctoral Program Research Foundation of Southwest University of Science and Technology (No. 11zx7129). The author is very grateful to both reviewers for carefully reading this paper and for their comments.

Received: 21 December 2012 Accepted: 17 March 2013 Published: 8 April 2013

\section{References}

1. Agarwal, RP, Deng, S, Zhang, W: Generalization of a retarded Gronwall-like inequality and its applications. Appl. Math. Comput. 165, 599-612 (2005)

2. Choi, SK, Deng, S, Koo, NJ, Zhang, W: Nonlinear integral inequalities of Bihari-type without class H. Math. Inequal. Appl. 8, 643-654 (2005)

3. Pachpatte, BG: Inequalities for Differential and Integral Equations. Academic Press, New York (1998)

4. Deng, S, Prather, C: Generalization of an impulsive nonlinear singular Gronwall-Bihari inequality with delay. JIPAM. J. Inequal. Pure Appl. Math. 9, Article ID 34 (2008)

5. Furati, KM, Tatar, NE: Power-type estimates for a nonlinear fractional differential equation. Nonlinear Anal. 62, 1025-1036 (2005)

6. $\mathrm{Ma}, \mathrm{QH}$, Yang, EH: Estimations on solutions of some weakly singular Volterra integral inequalities. Acta Math. Appl. Sin. 25, 505-515 (2002)

7. $\mathrm{Ma}, \mathrm{QH}$, Yang, EH: Bounds on solutions to some nonlinear Volterra integral inequalities with weakly singular kernels. Ann. Differ. Equ. 27(3), 283-292 (2011)

8. McKee, S: The analysis of a variable step, variable coefficient linear multistep method for solving a singular integro-differential equation arising from the diffusion of discrete particles in a turbulent fluid. J. Inst. Math. Appl. 23, 373-388 (1979)

9. McKee, S, Tang, T: Integral inequalities and their application in numerical analysis. Fasc. Math. 308(23), 67-76 (1991)

10. McKee, S, Tang, T, Diogo, T: An Euler-type method for two-dimensional Volterra integral equations of the first kind. IMA J. Numer. Anal. 20(3), 423-440 (2000)

11. Mazouzi, S, Tatar, NE: New bounds for solutions of a singular integro-differential inequality. Math. Inequal. Appl. 13, 427-435 (2010)

12. Medved', M: On singular versions of Bihari and Wendroff-Pachpatte type integral inequalities and their application. Tatra Mt. Math. Publ. 38, 163-174 (2007)

13. Henry, D: Geometric Theory of Semilinear Parabolic Equations. Lecture Notes in Math., vol. 840. Springer, New York/Berlin (1981)

14. Sano, H, Kunimatsu, N: Modified Gronwall's inequality and its application to stabilization problem for semilinear parabolic systems. Syst. Control Lett. 22, 145-156 (1994)

15. Ye, HP, Gao, JM, Ding, YS: A generalized Gronwall inequality and its application to a fractional differential equation. J. Math. Anal. Appl. 328, 1075-1081 (2007)

16. Medved', M: A new approach to an analysis of Henry type integral inequalities and their Bihari type versions. J. Math Anal. Appl. 214, 349-366 (1997)

17. $\mathrm{Ma}, \mathrm{QH}$, Pečarić, J: Some new explicit bounds for weakly singular integral inequalities with applications to fractional differential and integral equations. J. Math. Anal. Appl. 341, 894-905 (2008)

18. Cheung, WS, Ma, QH, Tseng, S: Some new nonlinear weakly singular integral inequalities of Wendroff type with applications. J. Inequal. Appl. 2008, Article ID 909156 (2008) 
19. Wang, $\mathrm{H}$, Zheng, K: Some nonlinear weakly singular integral inequalities with two variables and applications. J. Inequal. Appl. 2010, Article ID 345701 (2010)

20. Medved', M: Nonlinear singular integral inequalities for functions in two and $n$ independent variables. J. Inequal. Appl. 2000, 287-308 (2000)

21. Pachpatte, BG: Integral inequalities of the Bihari type. Math. Inequal. Appl. 5, 649-657 (2002)

doi:10.1186/1029-242X-2013-159

Cite this article as: Zheng: Bounds on some new weakly singular Wendroff-type integral inequalities and applications. Journal of Inequalities and Applications 2013 2013:159.

Submit your manuscript to a SpringerOpen ${ }^{\circ}$ journal and benefit from:

- Convenient online submission

- Rigorous peer review

- Immediate publication on acceptance

Open access: articles freely available online

- High visibility within the field

- Retaining the copyright to your article

Submit your next manuscript at $>$ springeropen.com 